JUSTYNA ŁUKASZEWICZ

ORCID: 0000-0003-2140-3610

Université de Wrocław

justyna.lukaszewicz@uwr.edu.pl

\title{
BEAUMARCHAIS TRADUIT ET ADAPTÉ POUR LE THÉÂTRE POLONAIS DU SIĖCLE DES LUMIÈRES
}

\section{INTRODUCTION}

Presque toutes les œuvres dramatiques de Pierre-Augustin Caron de Beaumarchais (1732-1799) ont été traduites et représentées en Pologne du vivant de leur auteur, à l'exception des parades et de La Mère coupable (1792), jouée à Varsovie en 1800 et publiée seulement en $1827^{1}$. Indirectement, Beaumarchais est présent dans le théâtre polonais du siècle des Lumières en tant qu'auteur de Tarare - opéra tragicomique comportant une critique de la tyrannie - représenté à Paris en 1787. Lorenzo Da Ponte en a créé la version italienne, qui a été exécutée à Vienne en 1788. Celle-ci a servi de modèle à l'Axur, król Ormus de Wojciech Bogusławski (joué à partir de 1793 $)^{2}$.

${ }^{1}$ [P.-A.] Caron de Beaumarchais, Matka wystęnna, czyli domowe troski familii Almawiwa. Dramma w pięciu aktach przez P. Caron de Beaumarchais Autora Komedyi Cyrulika Sewilskiego i matżeństwa Figara. Grana naypiérwszy raz w Paryżu dnia 26 Czerwca 1792 roku, [trad. J. Narzymski], Drukarnia Zymela, Wilno 1827.

${ }^{2}$ En ce qui concerne cette œuvre, le rapport des deux versions polonaises dues à Bogusławski (le manuscrit thêâtral créé vers 1822 et le texte publié en 1824) à la version italienne a été étudié dans : J. Łukaszewicz, « Continuité et rupture dans le domaine de la traduction théâtrale vers le polonais : Wojciech Bogusławski et son Axur », [dans :] Traduction et Rupture. La traduction comme moyen de communication interculturelle, textes réunis par M. Laurent, Éditions Numilog, Paris 2014, pp. 71-90. L'histoire de l'opéra de Salieri, dans ses différentes versions linguistiques, dont celle de Bogusławski, a été traitée dans : K. Lisiecka, «Ironiczne wyzwolenie. Axur. Król Ormus Antonio Salieriego i Wojciecha Bogusławskiego », [dans :] Europejski wiek osiemnasty — uniwersalizm myśli, 
L'objectif de cette analyse est d'approfondir la connaissance de la réception polonaise réservée à l'œuvre de Beaumarchais au siècle des Lumières. On étudiera les traductions présentées au public polonais du XVIII ${ }^{\mathrm{e}}$ siècle, préservées jusqu'à nos jours ${ }^{3}$, en tenant compte de leurs paratextes, surtout de ceux qui les accompagnent dans les éditions de l'époque ${ }^{4}$. Le corpus comprend la traduction d'Eugénie (Eugenia, 1778) ${ }^{5}$, une traduction (Cyrulik sewilski, albo ostrożność niepożyteczna, 1780) $)^{6}$ et une refonte (Zazdrość ukarana, albo Cyrulik warszawski, 1782) $)^{7}$ du Barbier de Séville et, finalement, la seule traduction du Mariage de Figaro (Dzień pusty albo Wesele Figara, 1786) ${ }^{8}$ qui nous reste des deux créées à l'époque.

On confrontera les versions polonaises aux originaux, pour chercher à découvrir - parmi les procédés de traduction/adaptation relevés — des traces d'affrontements politiques, sociaux ou culturels qui ont marqué la vie et l'œuvre de Beaumarchais. Il s'agit, en effet, d'un auteur qui « ne désarme jamais face à un adversaire ou un ennemi », d' " un battant, qui donne toute sa mesure dans les situations conflictuelles $»{ }^{9}$. Mon hypothèse est que les distances temporelles et culturelles ont probablement mené les traducteurs/adaptateurs à éliminer ou atténuer certains affrontements inscrits dans les textes de départ.

różnorodność dróg. Studia i materiały, M. Dębowski, A. Grześkowiak-Krwawicz, M. Zwierzykowski (dir.), Polskie Towarzystwo Badań nad Wiekiem Osiemnastym, Towarzystwo Naukowe "Societas Vistulana”, Warszawa-Kraków 2013, pp. 555-563.

3 On ne dispose plus de la version polonaise des Deux amis, ou le Négociant de Lyon (1770), Dwaj przyjaciele, czyli kupiec lugduński, pièce représentée en 1788 (voir : L. Bernacki, Teatr, dramat i muzyka za Stanisława Augusta, t. 2 : Notatki i studja, Wydawnictwo Zakładu Narodowego im. Ossolińskich, Lwów 1925, pp. 223-224).

${ }^{4}$ Les paratextes situés « dans l'espace du même volume » que le texte lui-même sont appelés par Gérard Genette « péritextes » (G. Genette, Seuils, coll. « Points Essais », Éditions du Seuil, Paris 2002, p. 11).

5 [P.-A. Caron de Beaumarchais], Eugenia, dramma w piaciu aktach proza z francuzskiego na polski język wyłożona, P. Dufour, Warszawa 1778, [dans :] Teatr polski czyli Zbiór komedii dram i tragedyi z naysławnieyszych autorow Francuzkich ttómaczonych, i przez aktorów Polskich na Teatrze Warszawskim granych, t. 4, P. Dufour, Warszawa 1779.

${ }^{6}$ [P.-A. Caron] de Beaumarchais, Cyrulik sewilski, albo ostrożność niepożyteczna, komedya we czterech Aktach pana de Beaumarchais, z francuzkiego przetłomaczona y na Teatrze Warszawskim grana, [trad. S. Kuszewski], P. Dufour, Warszawa 1780, [dans :] Teatr polski..., t. 20, 1794.

7 [P.-A. Caron de Beaumarchais], Zazdrość ukarana albo Cyrulik warszawski, komedya w pięciu aktach. Pierwszy raz grana na Teatrze Warszawskim w Miesiącu Wrześniu 1781 roku, P. Dufour, Warszawa 1782, [dans :] Teatr polski..., t. 31, 1794.

8 [P.-A. Caron] de Beaumarchais, Dzień pusty albo Wesele Figara, komedya w piaciu aktach pana de Beaumarchais po polsku przettumaczona, [trad. M. Wolski], P. Dufour, Warszawa 1786, [dans :] Teatr polski..., t. 20, 1794. [Dans la suite de cet article, les citations des versions polonaises des drames analysés proviennent des publications indiquées dans les notes 5-8].

9 G. Conesa, «Beaumarchais en scène », Cahiers de l'Association internationale des études françaises 42, 1990, pp. 151-152. 


\section{EUGÉNIE}

\section{PUBLICATION ET REPRÉSENTATIONS}

La première pièce de Beaumarchais, Eugénie (Comédie-Française, le 29 janvier 1767), a été traduite en huit langues ${ }^{10}$. La version polonaise, Eugenia, dont l'auteur reste inconnu, n'a été publiée qu'en 1778. Les représentations attestées datent de $1783^{11}$.

\section{PÉRITEXTES}

La page de titre de l'édition polonaise présente la pièce comme un drame en cinq actes traduit du français. L'auteur de l'original et le traducteur ne sont pas mentionnés.

Les péritextes de l'auteur ont été éliminés ou abrégés. En particulier, l'édition polonaise ne communique pas à ses lecteurs les idées de l'auteur sur le drame sérieux. On n'y trouvera pas l'Essai sur le genre dramatique sérieux dont Beaumarchais a fait précéder sa pièce et où il définit et défend ce genre nouveau comme « intermédiaire entre la Tragédie héroïque et la Comédie plaisante » ${ }^{12}$. D'après Beaumarchais, ce genre, " où il ne faut que persuader par le sentiment $»^{13}$, doit être « puisé dans nos mœurs $»^{14}$, présenter « la peinture touchante d'un malheur domestique $»^{15}$ et offrir un « sens moral à l'esprit $»^{16}$. L'auteur d'Eugénie se dit guidé dans son travail par l'excellent exemple du Père de famille de Diderot ${ }^{17}$. Dans la traduction polonaise, on a éliminé aussi le commentaire de l'auteur qui suit le premier jeu d'entracte et dans lequel il est question de l'importance de la vérité et de l'illusion que « l'action pantomime » contribue à créer.

\section{TEXTE}

L'action de cette version, comme celle de l'original, se déroule à Londres, et les noms des personnages sont les mêmes (baron Hartley, madame Murer, Betsy,

${ }^{10}$ V. Yvernault, « Figaromania in Europe: the circulation and appropriation of Beaumarchais's plays in the eighteenth century », [dans :] Moving Scenes. The Circulation of Music and Theatre in Europe (1700-1815), P.-Y. Beaurepaire, P. Bourdin, C. Wolff (dir.), Voltaire Foundation, Oxford University Studies in the Enlightenment, Oxford 2018, p. 158.

11 L. Bernacki, op. cit., t. 1 : Źródta i materjały, p. 264 ; t. 2 : Notatki i studja, p. 234.

12 Beaumarchais, Essai sur le genre dramatique sérieux, [dans :] Beaumarchais, Théâtre complet. Lettres relatives à son théâtre, édition établie et annotée par M. Allem et P. Courant, coll. «Bibliothèque de la Pléiade », Gallimard, Paris 1957, p. 5.

13 Ibidem, p. 5.

14 Ibidem, p. 11

15 Ibidem, p. 8.

16 Ibidem, p. 9.

17 Ibidem, p. 6. 
Robert) ou à peine changés, au moyen d'assimilation phonétique et graphique (Drink $\rightarrow$ Drynk) ou de l'introduction d'équivalents polonais attestés (Eugénie $\rightarrow$ Eugenia). Certains toponymes sont gardés, quoique généralement écrits « à la polonaise » (Suffolk-Street $\rightarrow$ ulica Soffok, «près du parc Saint-James » $\rightarrow$ « pre diu park sę żam »). Dans l'ensemble, la couleur locale anglaise est cependant moins riche. Elle a été atténuée par l'effacement des références à l'Angleterre telles que l'évocation des livres sterling ou du pays de Galles, ainsi que par l'élimination des formes d'adresse Miss et Milady.

En ce qui concerne les didascalies, et donc les détails de la conception scénique de l'auteur, le traducteur ne s'est embarrassé ni de l'« habillement des Personnages, suivant le Costume de l'état de chacun en Angleterre » (p. 23) ${ }^{18}$, minutieusement décrit par Beaumarchais, ni de la disposition exacte du salon où l'action se passe, pourtant considérée par ce dernier comme importante.

Dans la version polonaise d'Eugénie, la figure du père, affligé par les graves problèmes de ses enfants, reste la même. Il apprend que sa fille a été déshonorée par un faux mariage organisé par un homme d'un rang social plus élevé que le sien et que la carrière, voire même la vie de son fils, militaire, sont en danger car son colonel est devenu son ennemi. Tant en français qu'en polonais, la dynamique de la pièce est construite sur de nombreux affrontements liés à la vie familiale et sociale, aux problèmes de morale, aux questions d'honneur et d'amour : entre le père et la tante d'Eugénie, entre le frère et le faux mari d'Eugénie, entre Eugénie et son faux mari, entre ce dernier et son serviteur. Le traducteur a conservé aussi l'éloge de l'équité royale et le message égalitaire du dialogue dans lequel le Baron expose à sa sœur, Madame Murer, son projet de s'adresser au roi pour chercher auprès de lui l'appui dans ces affrontements avec ceux qui ont causé le malheur de ses enfants :

Le Baron : Ce que je lui dirai ? Je lui dirai : Sire... vous êtes père, bon père... je le suis aussi ; mais j'ai le cœur déchiré sur mon fils et sur ma fille. Sire, vous êtes humain, bienfaisant... [...]. Il m'écoutera, et j'ajouterai : Un suborneur est venu en mon absence violer notre retraite et l'hospitalité ; il a déshonoré ma fille par un faux mariage... Je vous demande à genoux, sire, grâce pour mon fils et justice pour ma fille.

Madame Murer : Mais ce suborneur est un homme qualifié, puissant.

Le Baron (vivement) : S'il est qualifié, je suis gentilhomme... Enfin, je suis un homme... Le roi est juste ; à ses pieds toutes ces différences d'état ne sont rien : ma sœur, il n'y a d'élévation que pour celui qui regarde d'en bas ; au-dessus tout est égal ; et j'ai vu le roi parler avec bonté au moindre de ses sujets comme au plus grand. (acte IV, scène 3, pp. 60-61)

Eugénie représente le genre créé notamment par Diderot et Beaumarchais qui - avec sa poursuite du vraisemblable, la place accordée à la condition, l'importance des relations familiales, le rôle des émotions, le non-recours au ressort comique, l'insistance sur la vertu, etc. - s'oppose aux conventions classiques.

18 Toutes les citations des versions originales des pièces de Beaumarchais ont été puisées dans : Beaumarchais, Théâtre complet... 
Il a été rapidement adopté par le Théâtre National de Varsovie qui, à l'époque, puisait très souvent son répertoire dans celui de la Comédie-Française. Bien que, dans l'édition polonaise, certains éléments textuels et péritextuels qui témoignent du projet théâtral de Beaumarchais ne soient pas rendus, ce qui y est essentiel au genre nouveau est apparu sur la scène polonaise. Dans sa présentation de la carrière de l'actrice Agnieszka Truskolaska, Wojciech Bogusławski la loue surtout pour le rôle principal dans cette pièce de Beaumarchais où son jeu a suscité dans le public des émotions profondes et inoubliables. En rappelant des scènes particulièrement émouvantes, l'homme de théâtre polonais définit la pièce « un modèle du genre $»^{19}$.

\section{LE BARBIER DE SÉVILLE - CYRULIK SEWILSKI}

\section{PUBLICATION ET REPRÉSENTATIONS}

Le Barbier de Séville, ou la Précaution inutile (Comédie-Française, le 23 février 1775) a été traduit en polonais par Stanisław Kuszewski ${ }^{20}$ comme Cyrulik sewilski, albo ostrożność niepożyteczna. La traduction a été publiée en 1780 et jouée en $1781^{21}$.

\section{PÉRITEXTES}

Le titre et le sous-titre ont été traduits littéralement.

Kuszewski a doté l'œuvre traduite d'une note où il s'exprime à la première personne:

Zazdrostka iest wzięte z słowa Francuzkiego jalousie, lubo nie używane, alem sobie pozwolił wziąć go dla tego, żeby było używane iak wiele innych. (I, 1, p. 7) 22 $^{22}$

Ce péritexte, rare dans les textes dramatiques publiés en Pologne à l'époque, remplit une fonction métapraxique et métalinguistique ${ }^{23}$. Le traducteur se présente comme créateur d'un mot polonais au moyen d'un emprunt au français. Il s'agit

19 «Drama Eugenia, w której Beaumarchais dał wzór pisania dzieł podobnego rodzaju », cité dans : Teatr Narodowy 1765-1794, J. Kott (dir.), Państwowy Instytut Wydawniczy, Warszawa 1967, p. 687.

${ }^{20}$ Ce fournisseur de répertoire peu connu a traduit également Don Juan de Molière (1781) et le livret de Marmontel pour l'opéra-comique Zémire et Azor (1782) — D. Ratajczakowa, Komedia oświeconych, Wydawnictwo Naukowe PWN, Warszawa 1993, p. 230.

${ }^{21}$ L. Bernacki, op. cit., t. 2, p. 206.

22 «Zazdrostka est un mot inspiré du mot français jalousie. Bien qu'il ne soit pas utilisé, je me suis permis de le prendre pour qu'il soit utilisé comme bien d'autres » (trad. J.Ł.).

${ }^{23}$ Cf. P. Sardin, « De la note du traducteur comme commentaire : entre texte, paratexte et prétexte », Palimpsestes 20, 2007, pp. 6-8 (<http://palimpsestes.revues.org/99> [consulté le 1.11.2019]). 
d'un mot-clé de la pièce, ayant deux sens complémentaires ${ }^{24}$ : l'un exprime le sentiment principal de l'antagoniste, l'autre se réfère à un objet lié à ce sentiment qui, en même temps, participe à la création de la couleur locale espagnole. En effet, comme Bartholo est jaloux de Rosine, elle ne peut se montrer au Comte que derrière sa jalousie, élément typique des maisons de Séville.

\section{TEXTE}

L'action de la comédie se passe toujours à Séville (malgré la conversion de pistol en czerwony złoty, monnaie polonaise de l'époque) et le nom du protagoniste mentionné dans le titre reste Figaro. Les autres personnages ont reçu des noms très proches de leurs modèles français (Almawiwa, Bartolo, Rozyna, Bazyli), à l'exception des domestiques de Bartholo, La Jeunesse et L'Éveillé, qui, contrairement aux tendances en vigueur dans le théâtre polonais de l'époque ${ }^{25}$, ont perdu leurs noms signifiants pour devenir Łukasz et Adam. Le Figaro de la version polonaise présente Lindor (le Comte déguisé) à Rozyna (Rosine) comme un jeune noble, alors que dans l'original, il est décrit comme « un jeune Bachelier» (II, 2, p. 184). Ce changement nuit à la logique de l'ensemble car l'intention du Comte (dans les deux versions de la comédie) est de se faire aimer comme un jeune homme d'origine modeste.

Le traducteur, qui nous a fait part d'une réflexion sur sa pratique, a commis cependant plusieurs erreurs de traduction, relatives notamment aux vêtements et à leurs couleurs dans la description des « Habits des Acteurs » : " grande fraise au cou » (p. 169) — «pukle wielkie aż na szyi » [grandes boucles jusqu'au cou] (p. 3) ; « culotte bleue et veste de même » (p. 170) — « spodnie i suknia biała » [...blanche...] (p. 6). Il a opéré aussi des amplifications, comme dans cette réplique de Figaro:

Hein, hein, quand il y aura des accompagnements là-dessus, nous verrons encore, Messieurs de la cabale, si je ne sais ce que je dis. (I, 2, pp. 172-173)

Otóż skończyłem ; a iak ieszcze przyidzie akkompaniament do tego, obaczemy, Mości Panowie kabaliści ieżeli nie wiem co mówię. Nawet i muzykanci mi tego nie zgania, chociaż tym Ichmościom cudza robota się nie podoba. Ale zazwyczay szewc drugiego szewca robotę gani ${ }^{26}$. (I, 2, pp. 9-10)

${ }^{24}$ Comment ne pas penser au roman La jalousie d'Alain Robbe-Grillet (1957) ?

25 En ce qui concerne la traduction d'anthroponymes dans le théâtre polonais du siècle des Lumières, voir : J. Łukaszewicz, «L'onomastique au théâtre : noms de personnages dans les drames de Franciszek Zabłocki et leurs modèles français », Romanica Wratislaviensia LIII, Wrocław 2006, pp. 99-100.

26 « Hé bien, j'ai fini, et quand il y aura encore des accompagnements là-dessus, nous verrons, Messieurs de la cabale, si je ne sais ce que je dis. Même les musiciens ne critiqueront pas mon travail, bien qu'à ces messieurs, le travail des autres ne leur plaise pas. Il est cependant habituel qu'un cordonnier désapprouve l'ouvrage d'un autre cordonnier » (trad. J.Ł.). J'utilise l'italique pour mettre en évidence un passage ajouté par le traducteur. 
Le fragment ajouté est une satire professionnelle dans le style de l'auteur traduit. En même temps, on observe un abaissement du niveau de langue par l'usage d'un proverbe.

D'un autre côté, il y a des omissions qui touchent, entre autres, l'image de l'Espagne, par l'élimination des toponymes la Manche et la Sierra-Morena dans le récit que Figaro fait de ses nombreuses aventures à son ancien-nouveau maître. Une autre liste abrégée est celle où on énumère les ennemis des gens de lettres. Des neuf éléments de la version originale, il ne reste que quatre. La vision satirique et l'image métaphorique dressées par Beaumarchais se trouvent appauvries par l'élimination de ceux qui se réfèrent aux insectes:

Voyant à Madrid que la république des lettres était celle des loups, toujours armés les uns contre les autres, et que, livrés au mépris où ce risible acharnement les conduit, tous les insectes, les moustiques, les cousins, les critiques, les maringouins, les envieux, les feuillistes, les libraires, les censeurs, et tout ce qui s'attache à la peau des malheureux gens de lettres, achevait de déchiqueter et sucer le peu de substance qui leur restait [...]. (I, 2, p. 175)

Widząc w Madrycie, że rzeczpospolita literalna była zgromadzeniem wilków zawsze uzbrojonych przeciwko sobie, i gdym widział, że wydani na wzgardę, którą na nich ściągało to śmieszne zażarcie, wszyscy krytykowie, zazdrośnicy, żurnalistowie, księgarze, i inni, którzy tylko przyszywaią się do skóry biednych uczonych, kończyli wyciskać i wysysać resztę soku, który im zostawał [...]. (I, 2, p. 15)

Les nombreuses réductions d'énumérations présentes dans l'original constituent une particularité de la méthode de traduction de Kuszewski. Il diffère sur ce point de son éminent collègue Franciszek Zabłocki, très actif, à la même période, comme traducteur-adaptateur du français et fournisseur du répertoire du Théâtre National de Varsovie, lequel a une prédilection pour les énumérations ${ }^{27}$.

Finalement, toujours dans le même échange entre Figaro et le Comte, le traducteur du Barbier a éliminé une allusion à l'insatisfaction des plaideurs dans les procès judiciaires, certainement une réminiscence des mauvaises expériences avec le système de justice vécues par l'auteur lui-même :

Figaro : Ah! comme je leur en garde, morbleu !

Le Comte: Tu jures! Sais-tu qu'on n'a que vingt-quatre heures au Palais pour maudire ses Juges? Figaro: On n'a que vingt-quatre heures au théâtre; la vie est trop courte pour user un pareil ressentiment ${ }^{28}$

Le Comte : Ta joyeuse colère me réjouit. [...] (I, 2, p. 175)

Figaro: Ah, do śmierci im tego nie zapomnę!

Hrabia: Twoy gniew zabawia mnie mocno. [...] (I, 2, p. 15)

Notons aussi la disparition du parallèle entre le tribunal et le théâtre (un des exemples de la forte métathéâtralité de cette pièce) et le manque d'équivalent

27 Cf. J. Łukaszewicz, Dramaty Franciszka Zabłockiego jako przekłady i adaptacje, Wydawnictwo Uniwersytetu Wrocławskiego, Wrocław 2006, pp. 281-284.

28 J'utilise l'italique pour mettre en évidence un passage omis par le traducteur. 
du mot « joyeuse » qui déforme la réplique du Comte au point de la rendre incompréhensible.

Dans cette comédie où Beaumarchais puise abondamment dans la tradition du théâtre comique pour la dépasser, des idées caractéristiques de l'époque sont inscrites dans des schémas et motifs traditionnels. Il s'agit en particulier de la caractéristique du serviteur et de sa relation avec son maitre. Le domestique est, certes, adjuvant indispensable pour que le maître obtienne la main de sa bien-aimée, mais il a aussi un passé intéressant, ainsi que plusieurs talents et compétences (notamment dans les domaines musical et littéraire) qui, d'une certaine manière, l'affranchissent de sa condition de dépendance. Ces traits de Figaro et ses affrontements avec le Comte sont en général rendus dans la traduction, avec cependant des limites comme celles mises en évidence ci-dessus.

La pièce contient aussi un "débat » idéologique dans lequel Rosine défend le siècle des Lumières face à son tuteur qui rejette ses acquis et valeurs, tous domaines confondus :

Bartholo : Qu'est-ce que la Précaution inutile?

Rosine : C'est une Comédie nouvelle.

Bartholo : Quelque Drame encore! Quelque sottise d'un nouveau genre !

Rosine : Je n'en sais rien.

Bartholo : Euh, euh ! les Journaux et l'Autorité nous en feront raison. Siècle barbare !...

Rosine : Vous injuriez toujours notre pauvre siècle.

Bartholo : Pardon de la liberté : qu'a-t-il produit pour qu'on le loue ? Sottises de toute espèce : la liberté de penser, l'attraction, l'électricité, le tolérantisme, l'inoculation, le quinquina, l'Encyclopédie, et les drames... (I, 3, p. 176)

Bartolo : Co to iest ostrożność niepożyteczna?

Rozyna : To iest komedya nowa.

Bartolo : Pewnie dramma iakie? iakie głupstwo nowego gatunku?

Rozyna : Prawdziwie nic nie wiem.

Bartolo : Eh! eh! dzienniki nam odpowiedzą za to. Wieku dziki!...

Rozyna : Zawsze WPan narzekasz na ten biedny wiek teraźnieyszy.

Bartolo : Proszę wybaczyć wolność moię. I coż nam uczynił dobrego? głupstwa wszelkiego rodzaju, wolność myślenia, attrakcyą, elektryczność, tolerancyą, inokulacyą, encyklopedyą, i drammata... (I, 3, p. 18)

Le traducteur polonais a fait de petites coupures dans les répliques de Bartolo, en éliminant deux éléments, l'Autorité et le quinquina, le premier pour lequel il était sans doute difficile de trouver un équivalent, et le deuxième anachronique car le quinquina était connu et appliqué dès le XVII ${ }^{\mathrm{e}}$ siècle. La traduction ne rend pas la nuance critique introduite par le terme tolérantisme, " nom donné par dénigrement au système de ceux qui croient qu'on doit tolérer dans un État toutes sortes de religions $»^{29}$, mais garde l'aspect métathéâtral : la méfiance vis-à-vis du nouveau genre - le drame (bourgeois).

29 Voir É. Littré, Dictionnaire de la langue française $(<\mathrm{https}: / / \mathrm{www}$. littre.org/definition/tol\%C $3 \%$ A9rantisme $>$ ). 


\title{
LE BARBIER DE SÉVILLE-ZAZDROŚĆ UKARANA, ALBO CYRULIK WARSZAWSKI
}

\author{
PUBLICATION ET REPRÉSENTATIONS
}

Il existe un autre avatar polonais du Barbier de Séville : Zazdrość ukarana, albo Cyrulik warszawski, pièce anonyme en 5 actes qui a été jouée en $1781^{30}$ et publiée en 1782.

\section{PÉRITEXTES}

Comme son sous-titre l'indique, l'action de cette comédie sur la jalousie punie (zazdrość ukarana) se passe à Varsovie (cyrulik warszawski = 'barbier varsovien').

\section{TEXTE}

La plus grande surprise qui attend les lecteurs/spectateurs qui y chercheraient des correspondances avec la fameuse pièce de Beaumarchais est que le barbier du titre est le tuteur de la fille qu'il veut épouser sans en être aimé. Par contre, le nom de la fille en question, Eugenia (Eugénie), fait penser à la première pièce de l'auteur français (publiée en polonais quatre ans avant). Trois personnages portent des noms signifiants : Zgrzęcki (cet anthroponyme caractérise le barbier-tuteur comme grincheux), son apprenti Brzytwa ('Rasoir') et Filutowicz (filut = escroc, trompeur), ancien domestique de Walery (Valère), l'amoureux d'Eugénie.

L'auteur polonais a introduit de nouveaux motifs, absents de la comédie de Beaumarchais : le tuteur a un frère, Erast, qui, lui aussi, veut épouser Eugenia ; Filutowicz déclenche un incendie pour détourner l'attention du tuteur-cerbère. On trouve quand même dans ce texte de nombreux motifs empruntés au Barbier de Séville : un tuteur jaloux, un amoureux secondé par un ancien domestique rusé, rencontré dans la deuxième scène de la pièce, la vie aventureuse de ce serviteur. En effet, il a été recommandé par son ancien maître à un fils de châtelain qui s'est révélé peu fiable. Manquant d'argent, il s'est fait tertiaire bernardin, mais les moines l'ont chassé pour s'être enrichi de manière malhonnête. Arrivé à Varsovie, il a vite dépensé son argent en compagnie de parasites. Il a décidé alors d'écrire des poèmes et finalement des pièces de théâtre, sans succès. Son curriculum vitae ressemble donc à celui de Figaro.

Dans la comédie polonaise, la musique est moins importante ; cependant Eugenia chante en jouant de la harpe et c'est une sérénade dans la rue qui détourne l'attention du vieillard tyrannique. Pareillement, l'auteur polonais exploite

${ }^{30}$ L. Bernacki, op. cit., t. 2, p. 320. 
les procédés métathéâtraux à la manière de Beaumarchais, mais avec moins de régularité. Par exemple, il a choisi pour Walery une autre identité d'emprunt : il se présente dans la maison de Zgrzęcki déguisé en tailleur. L'auteur polonais ajoute à cette scène un effet comique typique des adaptations de Zabłocki, à savoir l'introduction d'une langue étrangère ${ }^{31}:$ ici, le personnage mélange le polonais et l'allemand (III, 6).

La satire sociale est, dans la comédie polonaise, très restreinte par rapport à la pièce française, mais on trouve des flèches satiriques contre les médecins (intéressés) et même une série d'anecdotes à leur propos, en particulier sur la pratique abusive de la saignée (I, 2 ; III, 1). Le tuteur de la comédie polonaise ne s'indigne pas contre son siècle et ses inventions (tolérance, inoculation, électricité, drame), mais contre les romans, nocifs particulièrement aux jeunes filles (I, 3). Dans les deux comédies, la critique vise l'oppression de la femme par la tyrannie des barbons.

Le titre de la comédie polonaise met au centre non pas le domestique du projet révolutionnaire de Beaumarchais, mais le vieux tuteur tyrannique, auquel l'auteur polonais a attribué le métier de Figaro. Filutowicz (nom de domestique utilisé dans d'autres comédies polonaises de l'époque ${ }^{32}$ ) prend la place du Figaro français. C'est, par exemple, lui qui a le dernier mot de la comédie, par une réplique très proche de celle du Barbier de Séville, mais plus drastique et dépourvue de fonction métathéâtrale:

Figaro : Faute de sens. Mais soyons vrais, Docteur ; quand la jeunesse et l'amour sont d'accord pour tromper un vieillard, tout ce qu'il fait pour l'empêcher peut bien s'appeler à bon droit La Précaution inutile. (IV, 8, p. 230)

Filutowicz : [...] Młodym zostawmy miłość, starzy o śmierci myśleć powinni. [Laissons l'amour aux jeunes, les vieux devraient penser à la mort (trad. J.Ł.)] (V, 9, p. 137)

Filutowicz, qui n'a gardé que la première syllabe du nom de Figaro, représente bien la relation entre Le Barbier de Séville et Le Barbier de Varsovie. Il est tout aussi rusé et expérimenté que le Figaro de Beaumarchais et il remplit de manière semblable son rôle d'adjuvant du jeune amoureux, mais il se trouve renvoyé à la fonction du valet traditionnel, notamment par l'introduction du motif de l'amour parallèle des domestiques : en effet, Filutowicz fait la cour à la soubrette Reginka (IV, 1). Il a perdu plusieurs des traits qui placent Figaro à la croisée de diverses traditions littéraires et théâtrales et en font un protagoniste des Lumières. Par de nombreuses touches qui l'éloignent du Barbier de Séville, la comédie polonaise s'inscrit dans le courant de la « comédie de mœurs varsovienne » qui prospère dans les années 1780 .

31 Voir J. Łukaszewicz, Dramaty..., pp. 293-296.

32 Dans Zabobonnik (1781) de Franciszek Zabłocki, une adaptation du Superstitieux de JeanAntoine Romagnesi, et dans Egarz (1780) d'Ignacy Krasicki. 


\section{LE MARIAGE DE FIGARO}

\section{PUBLICATION ET REPRÉSENTATIONS}

Le Mariage de Figaro (Comédie-Française, le 27 avril 1784) a été représenté pour la première fois en Pologne, en français, le 22 décembre 1785, au Château Royal de Varsovie, en présence du roi (qui avait assisté aussi aux répétitions) et avec des acteurs illustres, aristocrates liés à la cour royale, de nationalités diverses : la duchesse Karolina de Nassau-Siegen (née Gozdzka), la comtesse Maria Teresa Tyszkiewiczowa (nièce de Stanislas-Auguste), Alojzy Fryderyk Józef von Brühl, Franciszek Woyna, chambellan du roi, Joseph de Maisonneuve, le comte Stepan Stepanovitch Apraxine ${ }^{33}$. Il est possible que les exemplaires utilisés aient été ceux que Beaumarchais a fait parvenir au roi de Pologne au printemps de 1785 alors que, emprisonné à Saint-Lazare, il cherchait la protection de souverains ${ }^{34}$.

En polonais, la pièce a été jouée d'abord à Vilnius, le 8 mai 1785 (fête du roi), ensuite à Varsovie, le 17 décembre de la même année. La traduction de Mikołaj Wolski (1762-1802), lui aussi chambellan de Stanislas-Auguste et son homme de confiance ${ }^{35}$, a été publiée l'année suivante. La version de Franciszek Zabłocki (probablement utilisée à l'époque au théâtre), ne nous est pas parvenue. En 1791, Wolski a évoqué, dans un poème, l'accueil qui a été réservé à sa traduction. Avec auto-ironie, il dit avoir été poussé à traduire cette pièce par des amis, il traite de folie la décision de publier le fruit de son travail et il compare sa version que personne ne lit à d'autres que le public connaît par cœur ${ }^{36}$.

La réception polonaise du chef-d'œuvre de Beaumarchais a démarré bien plus rapidement que dans le cas des pièces précédentes, mais, par rapport à son

33 A. Jendrysik, « Premiera Mariage de Figaro w Polsce », Przeglad Humanistyczny 3, 1959, pp. 177-181. Les conclusions de cet auteur corrigent ce qui a été établi par Ludwik Bernacki, selon lequel la pièce aurait été jouée à Varsovie, en français, en 1783 (c'est-à-dire avant la première parisienne), au théâtre de société de la duchesse de Nassau, à l'endroit nommé Dynasy (L. Bernacki, op. cit., t. 2, p. 240).

34 Extrait de la lettre de Ruault et Beaumarchais à La Hogue du 14 mars 1785 : « Vous êtes prié de la part de M. le Prince de Nassau qui m'en a chargé hier, d'adresser par le courrier à S.M. Le Roy de Pologne à Varsovie deux exemplaires du Mariage de Figaro broché, dont I. sous bande à Mlle la Princesse de Nassau, qui est actuellement à la Cour de S.M. Polonaise » (publié dans : G. and M. von Proschwitz, «Beaumarchais et le Courrier de l'Europe », Studies on Voltaire and the Eighteenth Century 273-274, 1990, vol. 2, pp. 874-875; cité dans : V. Yvernault, op. cit., p. 165).

35 Teatr Narodowy..., p. 261.

36 « Gdy wielu przekładało Figara wesele, I mnie też namówili moi przyjaciele.

Na honor! nie wiem, skąd mi szaleństwo się wzięło,

Żebym i ja też moje wydrukował dzieło.

Spotkała mnie nadgroda dudkom przyzwoita -

Tych na pamięć umieją, a mnie nikt nie czyta... »

(Teatr Narodowy..., p. 428)

Romanica Wratislaviensia 67, 2020

(C) for this edition by CNS 
accueil dans d'autres pays européens, la vitesse et l'intensité de cette réception n'ont rien d'exceptionnel. Par exemple, l'œuvre a été publiée en allemand quatorze fois en $1785^{37}$.

La presse polonaise a précédé et accompagné la réception théâtrale. En 1784, le mensuel varsovien Pamiętnik Historyczno-Polityczny informe ses lecteurs du succès du Mariage de Figaro à Paris. En 1785, la même revue et le journal Gazeta Warszawska publient des articles sur l'emprisonnement et la libération de Beaumarchais, ainsi que sur les réparations reçues par le célèbre dramaturge pour cette injustice. On y donne le nombre de représentations parisiennes qui ont suivi la première du Mariage (72) et on y rapporte les propos de l'auteur, qui aurait publié cette pièce malgré les menaces de féroces opposants présentés métaphoriquement comme des lions et des tigres ${ }^{38}$.

\section{PÉRITEXTES}

La page de titre de Dzień pusty albo Wesele Figara affiche le nom de l'auteur et la traduction des deux vers mis en exergue dans la comédie originale ( « En faveur du badinage, / Faites grâce à la raison ») que l'on retrouve dans le vaudeville final : " Przez wzgląd na dzieło ucieszne / Pofolguycie Rozumowi ». Dans sa préface (Przedmowa tlumacza), le traducteur présente la pièce comme une continuation du Barbier de Séville et comme une œuvre très bien accueillie en Europe. L'intérêt qu'elle suscite est dû aussi bien aux qualités de l'intrigue qu'au succès rencontré à Paris et aux démêlés judiciaires de l'auteur. Wolski défend aussi la langue polonaise, se dit modestement conscient de l'existence d'une autre traduction et conteste l'avis des critiques persuadés que c'est une pièce inadaptée à la Pologne au motif que les mœurs n'y sont pas aussi corrompues. Il soutient qu'il est bon qu'une pièce raille ce qui n'a pas existé pour que cela n'existe jamais. Pour finir, il présente les circonstances de la première de Vilnius, ce qui lui fournit l'occasion de rendre hommage au roi de Pologne.

Dans le même paratexte, le traducteur expose sa stratégie et ses procédés. Il s'agit, d'après Wolski, d'une traduction non littérale, qui tient compte plutôt de la langue d'arrivée que des mots de l'auteur. Le traducteur dit avoir observé comment va le monde, suivi les conseils des connaisseurs et de bons acteurs, adapté, éliminé, ajouté et corrigé inlassablement.

Dans le péritexte qui correspond à « Caractères et habillement de la pièce », Wolski remplace l'éloge de l'acteur Molé (interprète du rôle d'Almaviva) par celui de Wojciech Bogusławski (qui a joué ce rôle lors de la première à Vilnius), un homme de théâtre polyvalent, comparé à Molière. Contrairement à l'original, la version de Wolski ne mentionne pas les autres acteurs. Dans la présentation de

\footnotetext{
37 V. Yvernault, op. cit., p. 159.

38 Teatr Narodowy..., pp. 561-562.
} 
Zuzanna (Suzanne), le traducteur intègre la traduction du fragment de la Préface de Beaumarchais, " singulièrement belle », consacré au caractère de la camériste. La troisième intervention significative du traducteur dans ce paratexte auctorial est son observation critique à propos de Grippe-Soleil (Słońco-Piekos), personnage qu'il juge inutile. Il est vrai que cet emploi de paysan a été restreint par le traducteur qui n'a pas rendu son idiolecte et donc sa distinction sociale et sa fonction comique.

Outre la préface, Wolski a doté sa traduction de trois notes. Elles ont toutes un caractère métapraxique. Dans la première (p. 61), le traducteur signale une coupure qu'il a opéré : en effet, il n'a traduit que la moitié de la romance chantée par Chérubin. Dans la deuxième (p. 141), il explique deux sens du mot français pâté (terme culinaire et tache d'encre) qu'il croit avoir bien rendus par l'ethnonyme polonais żyd ('juif', mais aussi 'tache d'encre') ${ }^{39}$. Il ajoute que ce passage de l'original est aussi une allusion au fait que Beaumarchais a été accusé d'une tentative de corruption du tribunal avec de l'argent dissimulé dans un pâtét0 ${ }^{4}$. Il était donc conscient que l'auteur français réglait aussi ses propres comptes avec la justice.

Dans la troisième (p. 15), il explique pourquoi une partie importante de la scène 16 de l'acte III n'a pas été traduite : il a pensé que ce qui n'avait pas été joué à Paris, ne le serait pas en Pologne. Effectivement, les Comédiens Français ont retranché, au regret de l'auteur ${ }^{41}$, le discours véhément de Marceline où elle décrit la condition difficile des femmes et prend leur défense, en attaquant les hommes pour leur hypocrisie. Cet affrontement où la comédie vire au drame n'est pas nécessaire à l'intrigue, au contraire de l'affrontement entre Figaro et le Comte. Celui-ci se déroule en plusieurs étapes, dont le fameux monologue de Figaro, et Wolski en donne une traduction intégrale.

\section{TEXTE}

Dans le texte lui-même, le traducteur opère moins de modifications que sa préface n'en laisse attendre. L'allusion métathéâtrale à la comédie précédente est plus claire que dans l'original et — au prix d'un pléonasme — en accord avec le sous-titre de la traduction polonaise du Barbier de Séville : «malgré vos précautions » (I, 4, p. 263) devient « mimo twoich ostrożności niepożytecznych » ['malgré vos précautions inutiles'] (I, 4, p. 12). Il a parfois recours à la généralisation : par ex. « Je vais me retirer aux Ursulines [...]» (II, 19, p. 295) est rendu comme « Póydę do klasztoru » [Je vais me retirer au couvent] (II, 19, p. 85). Dans l'évocation de la musique paysanne, il remplace cornemuses par flutes (II, 20). Le Comte,

39 Il est étonnant que le traducteur appelle son équivalent « neutre », alors qu'il perpétue une association péjorative.

40 Wolski a pu se faire valoir par ex. de l'information contenue dans une note de l'édition publiée à Paris, chez les Marchands de Nouveautés, en 1785 : «On croit que ceci fait allusion à un pâté que M. de Goësman avait reçu dans la fameuse affaire de l'auteur de cette Piece » (p. 109).

41 Beaumarchais, Théâtre complet..., p. 784. 
avec une hache, semble plus brutal qu'avec « une pince à la main » (II, 16, p. 291). Le sens de quelques répliques est rendu avec recours à un proverbe, ce qui fait baisser le registre linguistique. Par d'autres petites touches, le traducteur introduit quelques éléments familiers au public polonais, tels que l'évocation d'une auberge dans le paysage campagnard ou des noms de chiens de chasse :

$\mathrm{Ah}$ ! voilà Monseigneur qui traverse à cheval le grand potager, suivi de Pédrille, avec deux, trois, quatre lévriers. (II, 1, p. 279)

Pan Hrabia pędzi konno przez Zwierzyniec, a za nim capay, łapay, ścinay, dołóż, dwa smycze Chartów. (II, 1, p. 52)

La comparaison de la traduction de Wolski avec l'original montre qu'il a apporté au texte de Beaumarchais des modifications assez nombreuses mais de détail. Comment a-t-il traité les nombreux affrontements entre les personnages qui constituent l'essentiel de cette pièce?

Il y a en particulier le litige qui oppose Marceline et Figaro. S'appuyant sur un contrat de prêt, la femme exige que Figaro l'épouse. Dans la scène de l'audience (III, 15 ; dans la traduction : III, 13) s'affrontent Bartholo, avocat de Marceline, et Figaro. En plus, tant l'avocat de la demanderesse que le Comte Almaviva, qui préside l'audience, cherchent à se venger de Figaro. La satire du monde judiciaire inscrite dans la scène dénonce la bêtise, le pédantisme, l'hypocrisie, la corruption et le ridicule des hommes de la loi. Les efforts du traducteur pour rendre la rude escarmouche de Figaro contre cette machine ne sont pas tous très adroits. Dans l'original, la dispute concerne plusieurs variantes possibles d'une phrase partiellement illisible à cause d'un pâté. Couvre-t-il et, ou ou où ? Avec ou sans virgule ? Les équivalents proposés sont moins clairs que la version originale. Dans la réplique fondamentale où Figaro oppose sa lecture du contrat à celle de Bartholo, le traducteur ajoute la trahison (zdrada) à la triade «malice, erreur, ou distraction » de l'original ; l'accusation est donc plus forte. Cependant, l'opposition ożenię z nia [je l'épouserai] / ożenienie [épousailles] n'est pas compréhensible sans le recours aux explications, assez tordues, dans d'autres répliques, ce qui diminue considérablement l'efficacité théâtrale de la scène entière :

Figaro : Qu'il y a, Messieurs, malice, erreur, ou distraction dans la manière dont on a lu la pièce ; car il n'est pas dit dans l'écrit : laquelle somme je lui rendrai, ET je l'épouserai ; mais : laquelle somme je lui rendrai, OU je l'épouserai ; ce qui est bien différent. (III, 15, p. 318)

Figaro : Nie zgadza się Prześwietny Sądzie. Jest zdrada, podstęp, pomyłka albo roztargnienie w sposobie czytania tey karty. Nie masz tam bowiem, iż się ożenię z nia, ale iż się ożenienie, co wcale iest co innego? (III, 13, p. 140)

Dans la version polonaise, il y a aussi, entre le docteur-avocat et Figaro, un échange de répliques plus longues que dans l'original où le traducteur a introduit des expressions latines. Absentes du texte de Beaumarchais, elles renouent avec la tradition moliéresque et s'inscrivent dans une forte tendance du théâtre polonais de l'époque. Elles caractérisent un personnage qui s'y prête bien car il représente 
en même temps la médecine et la justice, deux domaines traditionnellement visés par la satire théâtrale et attaqués dans l'œuvre de Beaumarchais.

\title{
CONCLUSIONS
}

Dans le corpus analysé, il y a trois traductions avec des adaptations ponctuelles (Eugenia, Cyrulik sewilski, Dzień pusty albo Wesele Figara) et une pièce inspirée de l'hypertexte, où le nom de Figaro/Barbier a été utilisé à des fins de marketing 42 (Cyrulik warszawski). Deux traductions sont dotées de paratextes du traducteur. De pièce en pièce, la réception de Beaumarchais en Pologne prend de l'ampleur : le temps passé entre la première française et la première polonaise se raccourcit, tandis que le nombre de versions de la même pièce, le volume de péritextes de traducteur et le nombre d'informations diffusées dans l'espace médiatique sont en croissance. La presse polonaise informe des affrontements de Beaumarchais avec le pouvoir à propos du Mariage de Figaro. La visibilité de l'auteur traduit et des traducteurs augmente.

La version polonaise d'Eugénie comprend les mêmes affrontements familiaux et sociaux que l'original. Dans celle du Barbier de Séville, les affrontements ont été légèrement atténués. Dans Le Barbier de Varsovie, comparé au Barbier de Séville, la satire sociale est restreinte, tandis que le personnage qui est un équivalent partiel de Figaro se trouve relégué au rang de valet traditionnel. Dans Dzień pusty albo Wesele Figara, l'affrontement révolutionnaire entre Figaro et le Comte est rendu, comme les autres (par ex. entre le Comte et la Comtesse), mais la scène de l'audience n'a pas un caractère aussi net que dans l'original et le traducteur a éliminé le discours « féministe » de Marceline. Par contre, dans l'espace paratextuel de l'édition polonaise de ce chef-d'œuvre, on trouve un écho des affrontements personnels de Beaumarchais.

\section{BEAUMARCHAIS TRANSLATED AND ADAPTED FOR THE POLISH THEATRE OF THE AGE OF ENLIGHTENMENT}

\author{
Summary
}

\begin{abstract}
Almost all the dramatic works by Pierre-Augustin Caron de Beaumarchais (1732-1799) have been translated and performed in Poland during his lifetime. The author of this article studies the $18^{\text {th }}$-century translations with their paratexts, comparing them with the original versions, in order to discover - among translation/adaptation procedures - traces of political, social, or cultural clashes that have marked Beaumarchais' life work and time. The corpus includes the translation of Eugénie (1778), a translation (1780) and a rewriting (1782) of The Barber of Seville and finally the only Polish version of The Marriage of Figaro (1786) that remains of the two created at the time.
\end{abstract}

42 Voir V. Yvernault, op. cit., p. 161. 
From play to play, the reception of Beaumarchais in Poland is growing: the time spent between the French première and the Polish one is shortening, while the number of translations, the volume of translator paratexts and the amount of information in the media space are increasing. The Polish version of Eugénie includes the same family and social clashes as the original. In the translation of The Barber of Seville, the clashes have been slightly mitigated. In The Barber of Warsaw, a play inspired by The Barber of Seville, social satire is limited, while the character being a partial equivalent of Figaro is relegated to the rank of traditional valet. In the translation of The Marriage of Figaro, all the confrontations, including that between Figaro and the Count, have been rendered, but the scene of the trial is not as sharp as in the original and the translator has eliminated Marceline's 'feminist' discourse. On the other hand, in the paratextual space of the Polish edition, there is an echo of Beaumarchais' personal clashes.

Key words: Beaumarchais, theatre translation, French drama in Poland, Enlightenment, paratexts. 